\title{
PAPER
}

\section{Routes to the preparation of mixed monolayers of fluorinated and hydrogenated alkanethiolates grafted on the surface of gold nanoparticles $\uparrow$}

\author{
Maria Şologan, ${ }^{a}$ Cristina Cantarutti, ${ }^{a}$ Silvia Bidoggia, ${ }^{a}$ Stefano Polizzi, ${ }^{b}$ \\ Paolo Pengo ${ }^{a}$ and Lucia Pasquato*a
}

Received 16th February 2016, Accepted 8th March 2016

DOI: $10.1039 / c 6 f d 00016 a$

The use of binary blends of hydrogenated and fluorinated alkanethiolates represents an interesting approach to the construction of anisotropic hybrid organic-inorganic nanoparticles since the fluorinated and hydrogenated components are expected to self-sort on the nanoparticle surface because of their reciprocal phobicity. These mixed monolayers are therefore strongly non-ideal binary systems. The synthetic routes we explored to achieve mixed monolayer gold nanoparticles displaying hydrogenated and fluorinated ligands clearly show that the final monolayer composition is a non-linear function of the initial reaction mixture. Our data suggest that, under certain geometrical constraints, nucleation and growth of fluorinated domains could be the initial event in the formation of these mixed monolayers. The onset of domain formation depends on the structure of the fluorinated and hydrogenated species. The solubility of the mixed monolayer nanoparticles displayed a marked discontinuity as a function of the monolayer composition. When the fluorinated component content is small, the nanoparticle systems are fully soluble in chloroform, at intermediate content the nanoparticles become soluble in hexane and eventually they become soluble in fluorinated solvents only. The ranges of monolayer compositions in which the solubility transitions are observed depend on the nature of the thiols composing the monolayer.

\section{Introduction}

In recent years, a significant interest in the development of gold nanoparticles protected by monolayers comprising fluorinated ligands has started to emerge ${ }^{1}$ and the now available strategies to improve their solubility, ${ }^{2,3}$ in addition to the

\footnotetext{
${ }^{a}$ Department of Chemical and Pharmaceutical Sciences and INSTM Trieste Unit, University of Trieste, via L. Giorgieri 1, 34127 Trieste, Italy. E-mail: lpasquato@units.it

${ }^{b}$ Department of Molecular Sciences and Nanosystems, University Ca' Foscari of Venezia, Via Torino 155/b, I30172 Venezia-Mestre, Italy

$\dagger$ Electronic supplementary information (ESI) available. See DOI: 10.1039/c6fd00016a
} 
remarkable examples of their properties, ${ }^{4-7}$ will likely stimulate future developments. Our previous studies, carried out on mixed monolayer gold nanoparticles (MMNPs) protected by blends of fluorinated (F-) and hydrogenated (H-) amphiphilic thiolates, demonstrated that phase segregation triggered by, inter alia, the reciprocal phobicity of the immiscible thiolates is operative and can be observed even when using less than $5 \%$ F-ligands.${ }^{8,9}$ Self-sorting of hydrogenated and fluorinated compounds is known to occur in the bulk phases, ${ }^{\mathbf{1 0}, 11}$ in supramolecular assemblies both at the micro- ${ }^{12,13}$ and nano-scale ${ }^{14-19}$ regime, or even at the molecular level..$^{\mathbf{2 0} 21}$ We believe that fluorophilic/fluorophobic interactions will also represent a powerful tool in the controlled formation of domains of well-defined morphology in the monolayer protected gold nanoparticles. The morphological features of mixed monolayers are central in determining the properties of nanoparticles such as their solubility behaviour and wettability; ${ }^{22}$ their interaction with biological membranes; ${ }^{23-25}$ their assembly properties ${ }^{26}$ and their catalytic activity. ${ }^{27} \mathrm{~A}$ large body of experimental evidence $^{28-35}$ and theoretical investigations ${ }^{36-40}$ provides a valuable framework for the understanding of the factors governing the formation of domains in the monolayer protected gold nanoparticles and other nanosized structures. ${ }^{\mathbf{4 1}}$ However, despite these outstanding achievements, there is a lack of comprehensive experimental data concerning the preparation of MMNPs. Even for well characterised MMNPs, the information on how differences in the molecular structures of the ligands used in the synthesis impact on the final composition of the monolayer is sparse. It is often assumed that the stoichiometric ratio of the ligands used in the synthesis is equal to the ratio on the ligand shell, ${ }^{29}$ and in some cases evidence does exist. ${ }^{35}$ In other cases, only the composition of the MMNPs is reported but not the ratio of the thiols used in the synthesis. ${ }^{42}$ In a different route to MMNPs that exploits the assembly of mixtures of thiols on the surface of dioctylamine-capped gold nanoparticles, ${ }^{43,44}$ it was also reported that the final composition of the monolayer reflects the initial composition of the ligand mixture. On the contrary, the place exchange reaction is known to be sensitive to the structure of the incoming thiols, with the equilibrium position depending on their length and steric bulk. ${ }^{45}$

Exploiting the fluorophobic/fluorophilic interactions between F- and H-thiolates may represent an advantageous additional tool to control the morphology of mixed monolayers. This approach, if successful, will allow us to bring the "chemical mismatch" between ligands to a maximum; hence maximizing the phase segregation behaviour. However, the preparation of F-/H-MMNPs is, at present, only partly explored. No comprehensive data on the synthetic conditions needed to introduce a given amount of fluorinated components into a monolayer of nanoparticles and their dependence on the thiol molecular structure are presently available. Moreover, the different bulkiness of F-versus $\mathrm{H}$-alkyl chains adds complexity to such an investigation. Based on these considerations we realised the urgency of fundamental analysis addressing this gap.

Understanding how the final monolayer composition depends on the initial composition of the reaction mixture in either the direct synthesis or in the place exchange reaction is thus instrumental to any approaches based on the use of Fand $\mathrm{H}$-thiols for the preparation of MMNPs. We reasoned that the only way to investigate this point in detail was to reduce the complexity of the systems to 
a minimum using simple fluorinated and hydrogenated model thiols of different lengths and steric bulks and exploring a wide range of monolayer compositions. Based on the experimental data, we discuss the correlation between the initial ratio of the thiols and the final composition of the monolayer in relation to the structure of the ligands. We also correlate the core size of the MMNPs to the nature of the thiols. Finally, we analyse the effect of the monolayer composition on the solubility properties of the MMNPs.

\section{Results and discussion}

\subsection{Design of the approach for the preparation of MMNPs}

In order to explore a broad range of conditions for the preparation of gold nanoparticles displaying mixed monolayers of $\mathrm{H}$ - and F-ligands and analyse how the monolayer composition depends on the ligand structure, we devised the library of simple thiols displayed in Fig. 1A. The fluorinated thiols we used are $1 \mathrm{H}, 1 \mathrm{H}, 2 \mathrm{H}, 2 \mathrm{H}^{-}$ perfluorooctanethiol (HF6) $\$$ and $1 H, 1 H, 2 H, 2 H$-perfluorododecanethiol (HF10) while the hydrogenated thiols are dodecanethiol (HC12), octanethiol (HC8), hexadecanethiol (HC16) and 3-methyldodecane-1-thiol (HbrC12). The thiols HF6, HC8, HC16 and HC12 are commercially available; thiol HbrC12 was synthesised according to Scheme S1 of the ESI $\uparrow$ while thiol HF10 was prepared according to literature procedures. ${ }^{46}$ The structural diversity of the thiols reported in Fig. 1A was conceived in order to analyse how structural parameters such as the thiol length, length mismatch and steric bulk influence the outcome of the syntheses.

The combination of thiols HC12 and HF10 in nanoparticles NP-C12/F10 or thiols HC8 and HF6 in nanoparticles NP-C8/F6 will allow an analysis of the conditions required for the preparation of MMNPs displaying $\mathrm{H}$ - and F-ligands of the same length. The combination of thiols HC12 and HF6 in nanoparticles NPC12/F6 or thiols HC16 and HF6 in nanoparticles NP-C16/F6, allows instead an analysis of the role played by the length mismatch (4 atoms and 8 atoms respectively) in the outcome of the syntheses. By combining thiols HbrC12 and HF6 in nanoparticles NP-brC12/F6 we will explore the effect of using a branched thiol, preventing the formation of a monolayer stabilised by van der Waals interactions, ${ }^{42}$ in association with a fluorinated thiol that is four atoms shorter. A cartoon representation of the MMNPs discussed is presented in Fig. 1B.

\subsection{Nanoparticle synthesis}

The preparation of the MMNPs NP-C16/F6, NP-C8/F6, and NP-brC12/F6 was achieved using direct synthesis following the Brust-Schiffrin procedure employing a blend of the H- and F-thiols. NP-C12/F6 and NP-C12/F10 were prepared using place exchange on nanoparticles bearing monolayers comprising hydrogenated thiolates only. For the preparation of the MMNPs by place exchange, narrowly dispersed NP-C12 were prepared using the method of Miyake. ${ }^{47}$ The use

\footnotetext{
$\$$ HF6 stands for $1 H, 1 H, 2 H, 2 H$-perfluorooctanethiol, with explicit reference to the sulfhydryl proton. The thiolate derived from $1 H, 1 H, 2 H, 2 H$-perfluorooctanethiol is reported as F6. All the thiols and thiolates in the text are named accordingly.

$\S$ NP-C12/F10 stands for mixed monolayer nanoparticles displaying the thiolates derived from thiols HC12 and HF10 in the monolayer. All of the nanoparticle systems described in the text are named accordingly.
} 
A
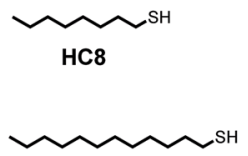

HC12
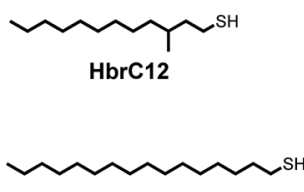

HC16

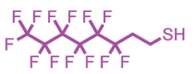

HF6

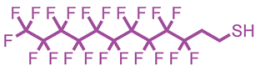

HF10

B

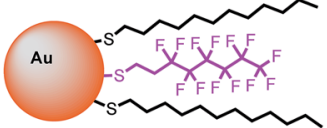

NP-C12/F6

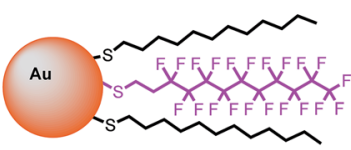

NP-C12/F10

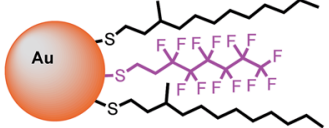

NP-brC12/F6

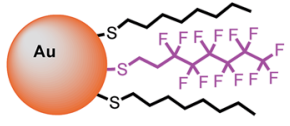

NP-C8/F6

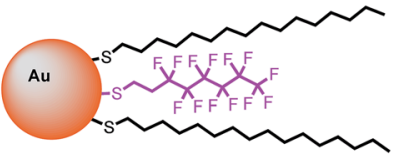

NP-C16/F6

Fig. 1 (Panel A) Library of the hydrogenated and fluorinated thiols used in this study. (Panel B) Structures of the mixed monolayer nanoparticles prepared by combining the thiols reported in panel $\mathrm{A}$.

of two different methodologies for the preparation of the MMNPs is particularly relevant because mass analyses show that mixed monolayers obtained by direct synthesis display little phase segregation, while ligand self-sorting seems to be more pronounced for the systems obtained by place exchange. ${ }^{48}$ The ratio between $\mathrm{H}$ - and F-thiolates in the monolayer was assessed by decomposing a small amount of the nanoparticles in the presence of excess iodine. The mixture of disulfides thus obtained was analysed using ${ }^{1} \mathrm{H}$ NMR and the ratio between the two thiolates present in the monolayer was determined by integration of the signals due to the methylene group in the alpha position to the sulfur atom.

The diameters of the nanoparticle cores were determined using TEM and the amount of organic material was assessed using thermogravimetric analyses. In some cases in both the direct synthesis and the place exchange reactions, two or three minor fractions of nanoparticles (all fractions from the same synthesis are indicated in the tables with the same lowercase letter) could be separated by exploiting their different solubilities in chloroform, hexane and/or hexafluorobenzene. All of these fractions have been completely characterised. The solubility properties of these nanoparticles are given by adding $(\mathrm{C}),(\mathrm{H})$ or $(\mathrm{F})$ to their designation, to indicate solubility in chloroform, hexane or hexafluorobenzene, respectively, as reported in Tables S1, S2, S6 and S8 of the ESI†.

\subsection{Analysis of the monolayer composition and composition-related properties}

Prior to presenting an analysis of the composition of mixed monolayers as a function of the relative amount of thiols in the initial reaction mixture, ${ }^{49}$ we find it useful to define the limiting behaviours expected under place exchange 
conditions or in direct synthesis. Let us consider the following cases: (i) the preparation of MMNPs by place exchange of thiol B from homoligand nanoparticles comprising thiolates A only. (ii) The preparation of MMNPs obtained by direct synthesis using a blend of thiols A and B. For the sake of simplicity, we shall consider the formation of a single population of nanoparticles.

Mixed monolayer obtained by place exchange. The relationship between the molar fraction of ligand $\mathrm{B}$ in the monolayer and its initial molar fraction in the reaction mixture can conveniently be represented by the plot in Fig. 2 . In this plot, the $x$-axis represents the initial molar fraction of thiol $\mathrm{B}$ in the reaction mixture, while the $y$-axis represents the final composition of the monolayer expressed as a molar fraction of the thiolate $\mathrm{B}$.

Three different limiting cases are theoretically possible depending on the relative affinity of thiols $\mathrm{A}$ and $\mathrm{B}$ and the thermodynamics of the monolayer formation. If we assume that the ligand $\mathrm{B}$ has a very high tendency to assemble in the monolayer and that this is much higher than that of thiol $\mathrm{A}$, in the place exchange reaction ligand $\mathrm{B}$ will displace ligand A completely. The amount of ligand B introduced into the monolayer is only limited by its initial molar fraction. This process will lead, eventually, to the complete conversion of the homoligand A-monolayer into a homoligand B-monolayer when the initial molar fraction of $\mathrm{B}$ is just 0.5 (red curve in Fig. 2); any further increase of the initial molar fraction of thiol B cannot produce further changes. A second limiting behaviour is obtained when no preference between ligand A and ligand B exists in forming the monolayer. The final compositions of the monolayers as a function of

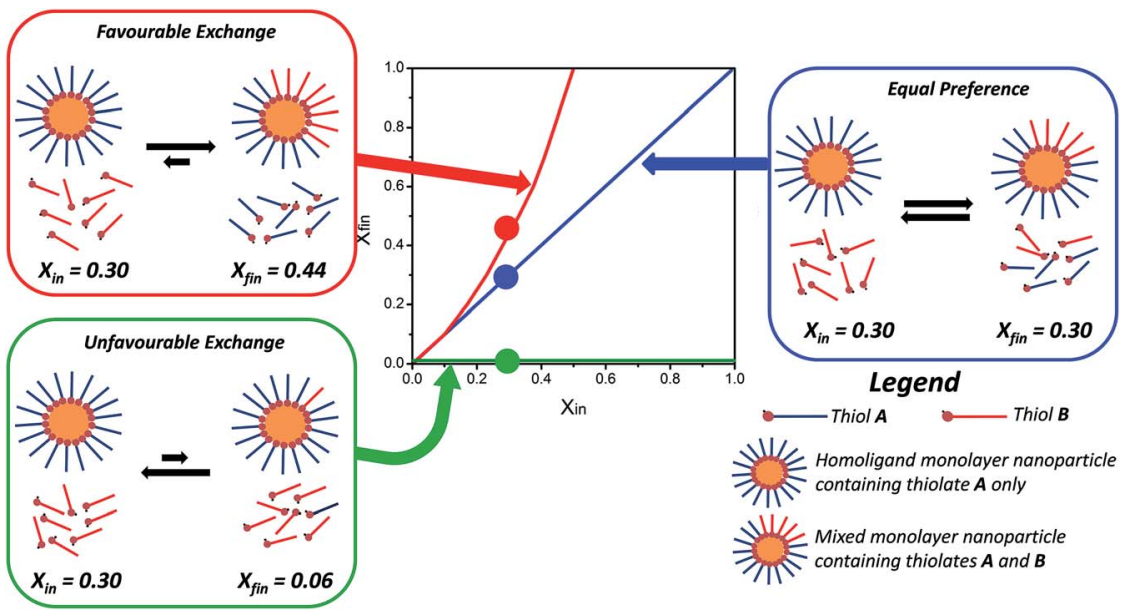

Fig. 2 Limiting cases theoretically possible in the place exchange between thiol B and homoligand nanoparticles featuring thiolate A only. If the grafting of thiol B is favoured (top left of the figure), complete consumption of thiol B can take place with displacement of an equal amount of thiolate A. In this case, the experimental data points will cluster along the red curve. If the grafting of thiol B is disfavoured (bottom left of the figure), only a small amount of exchange will be observed and the experimental data will be found close to the green line of the plot. If there is an equal preference for the grafting of ligands A or B (right hand side of the figure), the composition of the mixed monolayer will reflect the initial composition of the reaction mixture and the experimental data points will cluster along the diagonal of the plot. 
the initial compositions will thus cluster along the diagonal of the plot (blue line in Fig. 2). A third limiting case is the one pertaining to a situation in which the grafting of ligand B is strongly disfavoured (green line in Fig. 2). In this case, no B ligands will be found in the monolayer at any initial molar fraction.

Mixed monolayer obtained by direct synthesis. In the direct synthesis of MMNPs, the thiols are in excess with respect to the available grafting sites on the nanoparticle surface. $\uparrow$ This is a remarkable difference with respect to place exchange. If ligand B has a very high affinity for the monolayer and this is much higher than the affinity of thiol A, it is theoretically possible to end up with homoligand B-monolayers even if the initial molar fraction of thiol B is relatively small. In this case the experimental data points could be found either above the red line of Fig. 2 or close to it. On the other hand, if there is no preference for the grafting of the A or B thiolates, the experimental data points will cluster along the diagonal of the plot. If the grafting of thiol B is disfavoured the experimental points will be found beneath the diagonal or approaching the green line of the plot in Fig. 2.

Analysis of the experimental data for the synthesis of mixed monolayer nanoparticles. For nanoparticles NP-C12/F6 (Fig. 3A) the experimental data cluster along the diagonal of the plot, with some deviations only at very high initial loading of the fluorinated component. Hence, from a synthetic point of view, the preparation of nanoparticles NP-C12/F6 containing up to $50 \%$ of the fluorinated ligand is straightforward, since the initial molar fraction of the reaction mixture is retained in the final product. This indicates that there is essentially no energetic penalty or gain in introducing thiol HF6 into the monolayer of the preformed NP-C12. Instead, some energetic penalty, resulting in a less facile introduction of the F-ligand into the monolayer, occurs only when the initial molar fraction is very high. In some cases, the synthesis of nanoparticles NP-C12/F6 gives rise to a sub-population of nanoparticles with different solubility properties that are richer in the fluorinated component and that could be isolated (nanoparticles NP-C12/F6-i(H) and NP-C12/F6-k(H), Table S1). $\dagger$ The compositions of these systems are reported as open symbols in the plot of Fig. 3A.

Similarly, for nanoparticles NP-C12/F10 (Fig. 3B) the experimental data points tend to cluster close to or slightly above the diagonal. As in the previous case, in the preparation of nanoparticles NP-C12/F10, a small fraction of nanoparticles with monolayers very rich in the fluorinated component could be isolated (nanoparticles NP-C12/F10-a(H), NP-C12/F10-b(H) and NP-C12/F10-d(H), Table S2). $\dagger$ The composition of these nanoparticles is reported with open symbols in the plot of Fig. 3B. These data indicate a facile introduction of the fluorinated ligands into the monolayer of the nanoparticles even at a low molar fraction of the fluorinated component in the reaction mixture.

Upon increasing the length difference between the ligands, as in nanoparticles NP-C16/F6, a completely different behaviour was observed (Fig. 3C). In fact, the introduction of a few F-ligands into the monolayer of these nanoparticles proved

I The number of alkanethiolate chains on the surface of gold nanoparticles as a function of the total number of gold atoms is fairly well described by the equation: No. chains $\cong 2.5(N A u)^{-0.37}$. For nanoparticles with core size of $3.0 \mathrm{~nm}$ ( 976 gold atoms), the minimum theoretical Au/thiol ratio necessary to achieve passivation leaving no excess thiols is $\mathrm{Au} /$ thiols $=1 / 5$. For these calculations, we used the data reported in ref. 50 . 

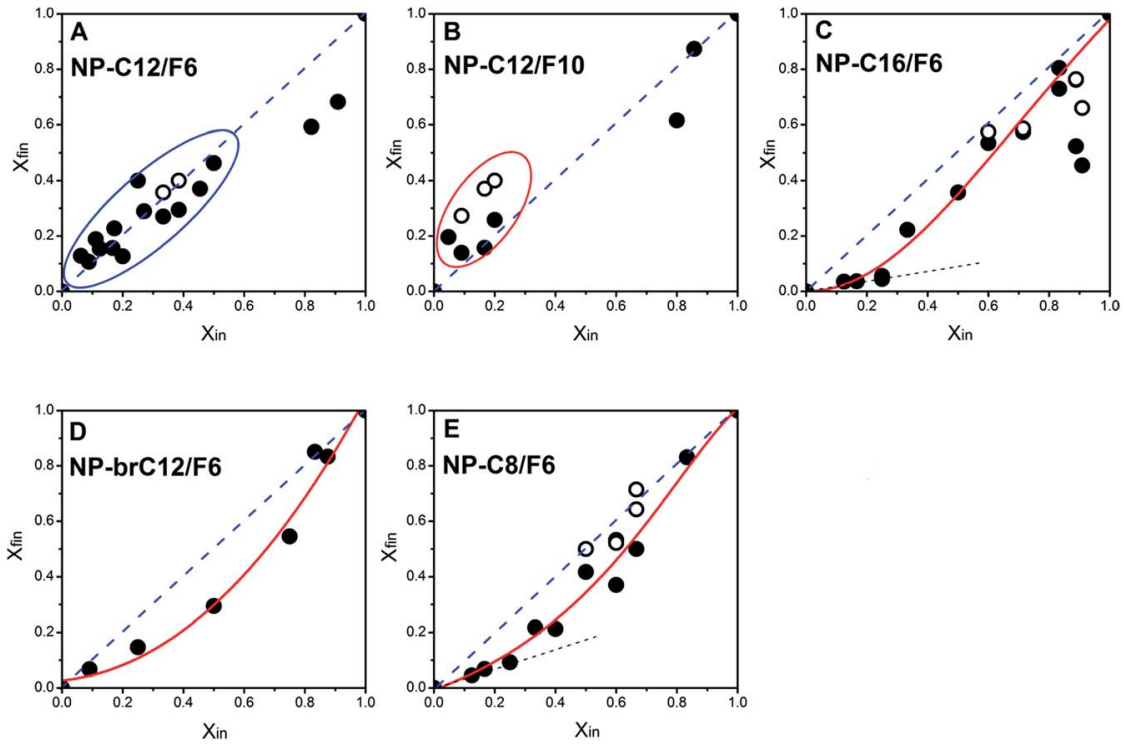

Fig. 3 (Panel A and B) Experimental data of the monolayer compositions for nanoparticles NP-C12/F6 and NP-C12/F10, respectively, as a function of the composition of the initial reaction mixture. (Panel $\mathrm{C}-\mathrm{E}$ ) Experimental data of the monolayer compositions for nanoparticles NP-C16/F6, NP-brC12/F6, and NP-C8/F6, respectively, as a function of the composition of the initial reaction mixture.

to be extremely unfavourable with a strong negative deviation from the diagonal of the plot in the region of a small initial fraction of the F-component. In these syntheses more than $20 \%$ of fluorinated ligand in the reaction mixture was necessary to achieve a mixed monolayer containing a mere $5 \%$ of fluorinated thiolates. After this threshold, however, the introduction of fluorinated ligands becomes more facile, with the experimental data points approaching the diagonal of the plot. Also in this case we observed the formation of sub-populations of nanoparticles richer in the fluorinated component, but, in contrast with NP-C12/ F6 and NP-C12/F10, this was observed only at initial molar fractions of the Fcomponent higher than $60 \%$; the compositions of these systems are reported with open symbols in the plot of Fig. 3C.

For nanoparticles NP-brC12/F6 (Fig. 3D) yet another behaviour appears. In this case the introduction of F-ligands remains unfavourable in all of the conditions explored. ${ }^{23}$ This is not unexpected because, by design and in analogy with literature evidence, these nanoparticles are believed to display a poorly organised monolayer. Notably for nanoparticles NP-brC12/F6, we could not identify subpopulations of nanoparticles with different fluorinated ligand content obtained in the same synthesis.

A somewhat intermediate behaviour was observed for nanoparticles NP-C8/F6 (Fig. 3E) where more than $20 \%$ of F-ligands in the reaction mixture was needed to achieve $10 \%$ of the F-thiolate in the final monolayer composition. After this threshold the introduction of F-ligands becomes more favourable, with the experimental data points slowly approaching the diagonal of the plot at higher molar fractions of HF6. In some cases, the preparation of nanoparticles NP-C8/F6 
yielded a small fraction richer in the fluorinated component when the initial composition of the reaction mixture contained more than $50 \%$ of the F-ligand. The compositions of these systems are reported with open symbols in the plot of Fig. 3E.

From these data it is clear that the formation of mixed monolayers comprising fluorinated thiolates may be favoured or disfavoured depending on the structure of the ligands and the degree of substitution that is achieved; relatively subtle structural changes impact considerably on the outcome of the syntheses. Most importantly, these data show that the final composition of the MMNPs cannot be a priori predicted on the basis of the composition of the reaction mixture, neither in the direct synthesis, nor in the place exchange reaction.

Effect of the fluorinated ligand loading on the nanoparticle size. It is well established by a large number of experimental results that in the Brust-Schiffrin synthesis, the size of the gold nanoparticles can be tuned by varying the initial gold/thiol ratio; the larger the ratio, the larger the resulting nanoparticles. ${ }^{50}$ There is also evidence that bulky thiols tend to favour the formation of smaller gold nanoparticles. ${ }^{51}$ Fluorocarbons have a cross-sectional area of $28.3 \AA^{2}$ while for hydrocarbons the molecular cross section is only $18.9 \AA^{2}{ }^{22}$ The fluorinated thiols used in this study are therefore much bulkier (1.5 times larger cross-sectional area) than the hydrogenated ligands, with the possible exception of the branched HbrC12. It is expected that the introduction of F-ligands into the monolayer of gold nanoparticles may result in systems of a small size. In the Brust-Schiffrin synthesis of MMNPs we indeed observed a monotonous decrease of the nanoparticle size by increasing the molar fraction of the F-component in the initial reaction mixture while maintaining a constant total gold/thiol ratio. This behaviour was found to be general, regardless of the difference in length between the F- and $\mathrm{H}$-ligands and the steric bulk of the hydrogenated thiols. The experimental data for the nanoparticles NP-C8/F6, NP-C16/F6 and NP-brC12/F6 are reported in Fig. 4A, B and C, respectively. The size contraction observed when a large molar fraction of the F-ligands is used clearly has a significant impact on the choice of the reaction conditions. In addition, it is also likely to have an impact on the organization of the monolayer since the morphology of mixed
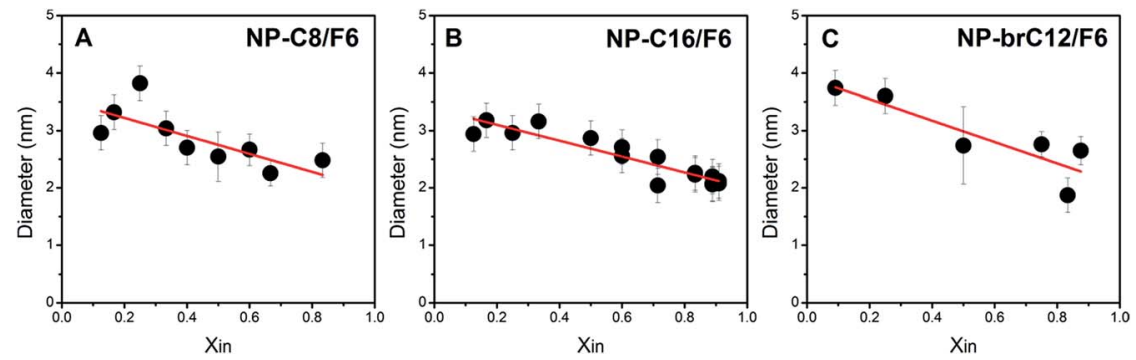

Fig. 4 Dependence of the nanoparticle core diameter on the initial molar fraction of the fluorinated ligand. Error bars represent the standard deviation of the average diameter measured using TEM analyses. In the case of multiple preparations with the same initial loading of the fluorinated component, the experimental points represent the average of the diameter and error bars represent their standard deviation. Panel A-C: experimental data of the diameters for nanoparticles NP-C8/F6, NP-C16/F6 and NP-brC12/F6 respectively. 
monolayers also depends on the nanoparticle curvature radius and in turn on the free volume available per chain.

Solubility behaviour of MMNP. The solubility properties of these nanoparticle systems are very informative and were found to vary according to the amount of fluorinated ligand in the monolayer and the structure of the fluorinated and hydrogenated thiolates. At low molar fractions of the fluorinated component, the nanoparticles were freely soluble in chloroform and methylene chloride. At an intermediate content of the fluorinated component, the nanoparticles were soluble in hexane, while at the higher molar fractions, they were soluble in fluorinated solvents only. In all of the cases, the solubility limit in the different solvents was higher than about $10 \mathrm{mg} \mathrm{mL}^{-1}$. To qualitatively analyse the solubility behaviour in relation to the monolayer composition, we found it convenient to use the solubility in the different solvents as a categorical variable and to plot this variable against the monolayer composition expressed as a molar fraction of the F-ligand (Fig. 5).

To the systems fully soluble in chloroform we arbitrarily assigned a score of 1 , the nanoparticle systems soluble in hexane were ranked with a score of 0.5 and those soluble in fluorinated solvents only (hexafluorobenzene was used throughout the study) were ranked with a score of zero. This approach allows the simple comparison of the solubility properties of a different set of nanoparticles. Nanoparticles NP-C12/F6 display a solubility transition, corresponding to the onset of solubility in hexane, when about $40 \%$ of the fluorinated thiolate is present in the monolayer; the same percentage was found for nanoparticles NP-C12/F10. A
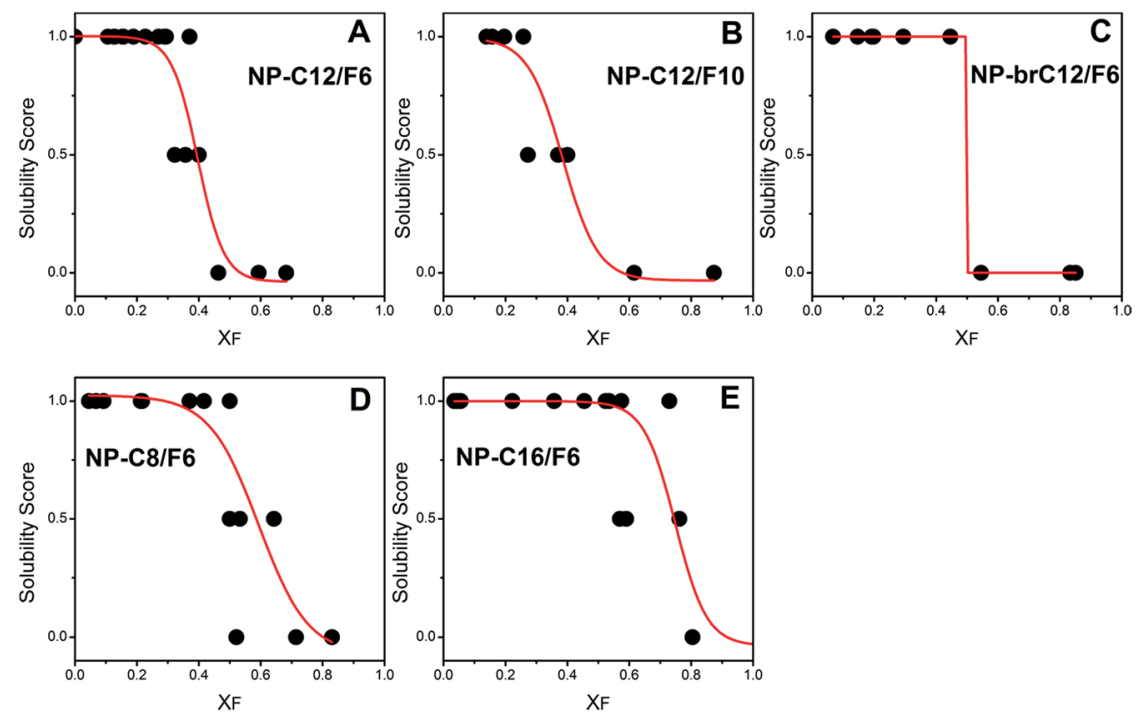

Fig. 5 Comparison of the solubility transitions for the MMNPs as a function of the molar fraction of the fluorinated component in the monolayer. The solubility is expressed according to the following score: score $=1$ is assigned to the nanoparticles that are soluble in chloroform. Score $=0.5$ is assigned to the nanoparticles that are soluble in hexane, score $=0$ is assigned to the nanoparticles that are soluble in hexafluorobenzene. Panel A-C: solubility scores for nanoparticles NP-C12/F6, NP-C12/F10 and NP-brC12/F6 respectively. Panel D-E: solubility scores for nanoparticles NP-C8/F6 and NP-C16/F6. 
significant difference was instead observed for nanoparticles NP-C16/F6 that remained fully soluble up to a molar fraction of fluorinated ligand of 0.8. For nanoparticles NP-brC12/F6, the transition occurred when the molar fraction of the F-ligand was 0.5; the same behaviour was found for nanoparticles NP-C8/F6.

\subsection{Discussion}

A rationalization of the experimental evidence presented above can be put forth by considering that the immiscibility of $\mathrm{H}$ - and F-ligands may lead to self-sorting of the two species and that the difference in their length and/or steric bulk may substantially contribute to the driving force of the self-sorting process.

Direct evidence of these phenomena have been already reported for mixtures of $\mathrm{H}$ - and F-ligands; ${ }^{9}$ further detailed studies addressing the influence of the ligand structure on the determination of the extent of clustering and the monolayer morphologies of the MMNPs presented here are ongoing in our lab. From a general point of view, if short fluorinated thiolates tend to cluster in a monolayer of longer hydrogenated ligands, the introduction of a very small number of F-ligands in the monolayer will be unfavourable. This is because introducing a few fluorinated ligands will decrease the number of van der Waals contacts between hydrogenated chains without offering a significant enthalpic gain derived from the establishment of fluorophilic interactions. In addition an unfavourable $\mathrm{H} / \mathrm{F}$ interface will be formed. At this stage the entropic gain due to the increased conformational mobility of the hydrogenated thiolates will also be minimal. Only when the amount of F-ligands exceeds a certain threshold should the introduction of more fluorinated ligands become favourable because of the increased number of fluorophilic interactions and the increased entropic gain associated with the conformational mobility of the longer hydrogenated thiolates. This is reminiscent of a cooperative process, where the (unfavourable) introduction of the first few fluorinated ligands generates the conditions for a more favourable assembly. We can clearly trace this phenomenon to the synthetic conditions explored for the preparation of nanoparticles NP-C16/F6 (Fig. 3C). In this case the introduction of up to $5 \%$ fluorinated ligands is strongly disfavoured, while after this threshold it becomes more favourable. The change in slope in the plot of Fig. 3C is consistent with the cooperative mechanism outlined above; in the first phase a few nucleation centres are formed that eventually evolve towards the growth of fluorinated domains. Nucleation and growth of alkanethiolate monolayers by displacement of weakly bound ligands on the surface of gold nanoparticles has indeed been reported. ${ }^{53}$ Our data may be taken as an indication that in the case of nanoparticles NP-C16/F6, 5\% F-ligand is sufficient to trigger the formation of fluorinated domains. This percentage is very close to the results of our previous studies showing that already at 5\% loading, domains are formed in mixed monolayers comprising amphiphilic $\mathrm{H}$ - and F-ligands. ${ }^{9}$ Another remarkable property of NP-C16/F6 is that up to $80 \%$ fluorinated ligand can be introduced without significantly affecting the solubility of the system. This implies that the Fligands cannot form large solvent exposed domains that would trigger particle aggregation. On the other hand, the data of Fig. 3C do suggest that domains indeed exist implying that these should be relatively small and/or shielded from the solvent and from the fluorinated domains of other nanoparticles by the longer H-ligands. 
For nanoparticles NP-C12/F10, the experimental data points cluster slightly above the diagonal of the plot of Fig. 3B indicating that the introduction of the Fligands into the monolayer is favourable even at low loadings of the fluorinated component. It should also be noted that for these nanoparticles we observed the formation of a second population of nanoparticles with a high fluorinated ligand content and different solubility properties even when only $10 \%$ of thiol HF10 was used in the place exchange reaction. Taken together, these data suggest a strong tendency of F10 to be assembled in the monolayer of nanoparticles NP-C12/F10. When, as in this case, there is no length mismatch between the thiolates, the enthalpic balance due to the loss of interactions between hydrogenated ligands and the establishment of fluorophilic interactions will be a significant contribution to the overall $\Delta G$ of reaction; a further (unfavourable) contribution to the $\Delta G$ of reaction will be the formation of an $\mathrm{H} / \mathrm{F}$ interface. Based on our experimental evidence, it is reasonable to think that for NP-C12/F10, clustering of ligands is likely to occur initially with the formation of small patches, eventually evolving towards larger compact domains, minimising in all cases the unfavourable formation of $\mathrm{H} / \mathrm{F}$ interfaces.

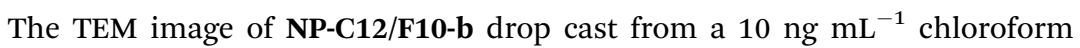
solution (Fig. 6) shows the formation of nanoparticle dimers and trimers accounting for $50 \%$ of the total population. As a comparison, the TEM image of NP-C12/F6-k(H), in the same conditions, shows $15 \%$ of dimers and trimers in the total nanoparticle population. This specific self-assembly pattern supports the existence of a well-defined anisotropy in the nanoparticle monolayer, consistent with the presence of large fluorinated and hydrogenated domains. The aggregation is likely due to the interaction of the fluorinated domains pertaining to different particles.

Given the absence of length mismatch between the two thiols HC12 and HF10, the observed behaviour is consistent with Glotzer's theoretical prediction of Janus nanoparticles.

As in the preceding case, the experimental data for nanoparticles NP-C12/F6 (Fig. 3A) indicate that there is essentially no energetic penalty or gain in

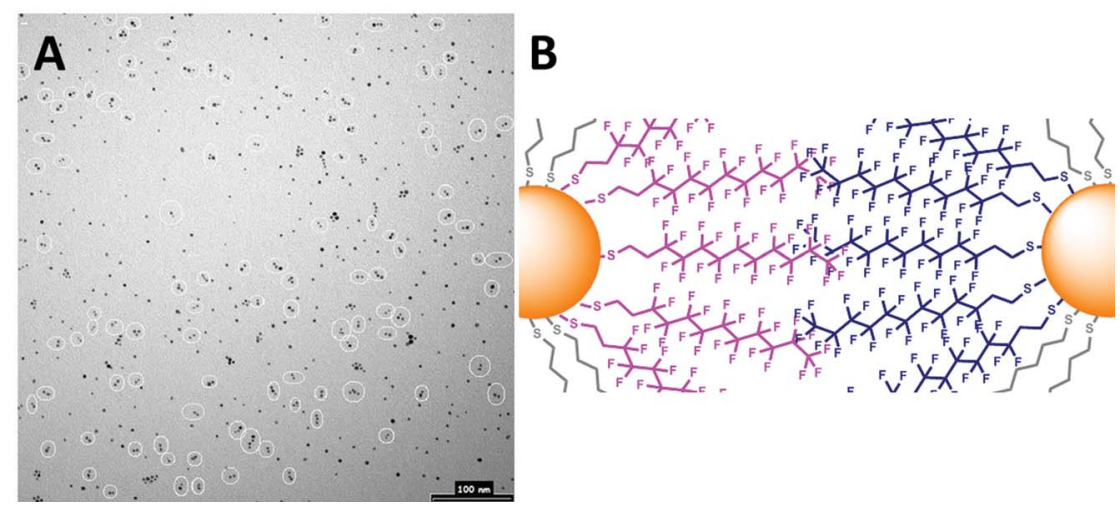

Fig. 6 (a) TEM image (magnification of $140 \mathrm{k} \times$ ) of nanoparticles NP-C12/F10-b drop cast from a $10 \mathrm{ng} \mathrm{mL}^{-1}$ chloroform solution. Dimers, trimers and some higher oligomers are circled in white. (b) Schematic representation of the interdigitation between fluorinated ligands pertaining to different Janus nanoparticles. 
introducing thiol HF6 into the monolayer of preformed NP-C12 up to a final composition of $50 \%$. Since, experimentally, the introduction of the fluorinated ligand was found to be easy even at very low loading, we expect the initial formation of small domains as in the case of NP-C12/F10. However, in contrast with the previous case, for nanoparticles NP-C12/F6 a dodecanethiolate unit is replaced by the $\mathbf{F 6}$ unit that is four atoms shorter. If clustering of the fluorinated ligands takes place, this geometrical mismatch should produce an increased conformational freedom for the hydrogenated ligands at the boundaries of the Fligand clusters. In this case domains with a large interfacial area to surface ratio could be expected.

The solubility properties of both nanoparticles NP-C12/F6 and NP-C12/F10 are in keeping with the formation of clusters of ligands, leading to the onset of low solubility already when only about $40 \%$ of the F-ligands are introduced into the monolayer (Fig. 5A and B respectively).

For nanoparticles NP-brC12/F6, the experimental data points cluster beneath the diagonal of the plot of Fig. 3D, suggesting an unfavourable assembly of the ligand $\mathbf{F 6}$ in the nanoparticle monolayer. Since in this case the branched nature of the brC12 thiolate hinders the formation of a compact monolayer, the formation of domains will be unlikely and a random distribution of the thiolates on the monolayer will result, in analogy to the observation of Stellacci and co-workers. ${ }^{42}$ The absence of fluorinated domains is consistent with the solubility properties of these systems that remain soluble in chloroform up to the introduction of $50 \%$ fluorinated component. Experimental evidence that is consistent with a random distribution of the thiolates is also the absence of sub-populations of nanoparticles, obtained in the same synthesis but displaying a different average content of F-ligands. Indeed these sub-populations are likely to be formed only if the introduction of F-ligands results in the formation of clusters in the monolayer. A somewhat intermediate behaviour is displayed by nanoparticles NP-C8/ F6; indeed, the graph of Fig. 3E shares some features with that obtained for nanoparticles NP-brC12/F6 and nanoparticles NP-C16/F6. This is peculiar since given the absence of length mismatch between the two thiols, the composition of the monolayer as a function of the initial molar fraction of the F-ligand should display a trend similar to that obtained for nanoparticles NP-C12/F10.

\section{Conclusions}

In this study we reported an analysis of the synthetic conditions for the preparation of MMNPs displaying fluorinated and hydrogenated ligands of different lengths and steric bulks. The nanoparticles were synthesised either by exploiting direct synthesis or by place exchange on preformed hydrogenated NPs. In most of the cases, the composition of the monolayer of MMNPs comprising fluorinated and hydrogenated ligands was found to be a non-linear function of the initial composition of the reaction mixture. The amount of fluorinated component introduced into the monolayer depends on the structures of the fluorinated and hydrogenated thiolates. The observed behaviours, including the deviations from linearity, are consistent with the tendency of the fluorinated and hydrogenated species to self-sort and with the role of the length mismatch in triggering the formation of domains by contributing to the overall thermodynamics of the monolayer assembly. The solubility properties of the nanoparticles are also 
consistent with the formation of fluorinated and hydrogenated domains in the monolayer of these systems. Taken together, the data presented here suggest that for nanoparticles NP-C16/F6, that display the largest difference in thiolate length among the systems explored, the formation of the mixed monolayer is dominated by the initial formation of small nucleation centres and the further growth of the domains is cooperative. In the case of nanoparticles NP-C12/F6 and NP-C12/F10, the formation of small domains is likely to occur, and to be favoured, even at very small loadings of the fluorinated component. For nanoparticles NP-brC12/F6 the experimental evidence fully supports the absence of any organization of the monolayer, in agreement with the observation of Stellacci and co-workers ${ }^{42}$ regarding the effect exerted by branched thiolates on the organization of mixed monolayer nanoparticles. Further specific experimental and theoretical investigations are in progress to assess the morphology of the monolayer of these MMNPs. Overall, the study presented here represents the first systematic approach aimed at defining a useful guideline for the design of synthetic strategies for the preparation of MMNPs. This work also shows that for MMNPs, the analysis of the monolayer composition as a function of the molar fraction of the reacting thiols used in the synthesis may provide information on the tendency of the thiolates to cluster on the surface of the nanoparticles.

\section{Experimental}

\subsection{Synthesis}

Synthesis of MMNPs. The synthesis of the MMNPs NP-C12/F6 and NP-C12/F10 was performed by place exchange from NP-C12 prepared according to a literature procedure. ${ }^{47}$ The synthesis of NP-C8/F6, NP-brC12/F6, and NP-C16/F6 was performed using the Brust-Schiffrin ${ }^{54}$ method with a mixture of hydrogenated and fluorinated thiols. The general procedure for the syntheses are outlined below; synthetic details are reported in Table S1 for NP-C12/F6, Table S2 for NP-C12/F10, Table S3 for NP-brC12/F6, Table S5 for NP-C8/F6 and Table S7 for NP-C16/F6. $\dagger$ Characterization data are presented in Table S1 for NP-C12/F6, Table S2 for NPC12/F10, Table S4 for NP-brC12/F6, Table S6 for NP-C8/F6 and Table S8 for NPC16/F6. $\dagger$

\subsection{General procedure for the place exchange reaction}

Nanoparticles NP-C12/F6 and NP-C12/F10. A solution of NP-C12 dissolved in DCM at a concentration of $2 \mathrm{mg} \mathrm{mL} \mathrm{mas}^{-1}$ weoxygenated and used for the synthesis. To the nanoparticles, a solution of fluorinated thiols in deoxygenated DCM (the proper amount is reported in Table S1 for NP-C12/F6 and Table S2 $\uparrow$ for NP-C12/F10) was added. The reaction mixture stirred at $40{ }^{\circ} \mathrm{C}$ in a pressure-tight screw-capped reaction vessel for three days. After this time the solution was concentrated to a small volume (about $5 \mathrm{~mL}$ ) and the nanoparticles were precipitated by addition of methanol. The supernatant was discarded and the precipitated nanoparticles were dissolved in $1 \mathrm{~mL}$ of $\mathrm{CHCl}_{3}$ and precipitated a second time by addition of methanol. The supernatant was discarded and the solid residue was dissolved in a small amount of $\mathrm{CHCl}_{3}$ and transferred to a centrifuge tube. The solvent was removed with the aid of a gentle argon stream and then the residue was washed with methanol $(4 \times 20 \mathrm{~mL})$ and acetone $(4 \times$ 
$20 \mathrm{~mL}$ ). To improve the purification process, the nanoparticles were dissolved in $\mathrm{CHCl}_{3}$, the solvent was removed under an argon flux, and the residue was washed with methanol $(4 \times 20 \mathrm{~mL})$ and acetone $(4 \times 20 \mathrm{~mL})$. The purified nanoparticles were subjected to selective extractions first with $\mathrm{CHCl}_{3}$ and afterwards with hexane. The insoluble material eventually present was tested for solubility in hexafluorobenzene. All of the fractions were characterised using ${ }^{1} \mathrm{H}$ NMR, UV-VIS, TGA, TEM. Characterization data are reported in Table S1 for NP-C12/F6 and Table S2 for NP-C12/F10. $\dagger$

\subsection{General procedure for the direct synthesis of nanoparticles NP-brC12/F6, NP-C8/F6 and NP-C16/F6}

A solution of tetraoctylammonium bromide (2.5 equivalents) in DCM was added to an aqueous solution of $\mathrm{HAuCl}_{4} \cdot 3 \mathrm{H}_{2} \mathrm{O}$ (1 equivalent) - see Table S3 for NPbrC12/F6, Table S5 for NP-C8/F6 and Table S7 for NP-C16/F6. $\dagger$ The mixture was vigorously stirred and the fading of the aqueous phase was observed while the organic phase turned orange. After the phase transfer was complete, a freshly prepared solution of the hydrogenated and fluorinated thiols in DCM was added to the reaction mixture. The concentration of the thiol solution and the volume used varied in the different syntheses. The total amount of thiol and their molar ratios are reported in Table S3 for NP-brC12/F6, Table S5 for NP-C8/F6 and Table S7 for NP-C16/F6. $\dagger$ The reaction mixture was left stirring at room temperature for 10 minutes and afterwards, a freshly prepared aqueous solution of $\mathrm{NaBH}_{4}$ was added under vigorous stirring; the time required for adding the $\mathrm{NaBH}_{4}$ solution is reported in Table S3 for NP-brC12/F6, Table S5 for NP-C8/F6 and Table S7 for NPC16/F6. $\uparrow$ The reaction mixture was left stirring for 18 hours at room temperature.

\subsection{General procedures for work-up}

Nanoparticles NP-brC12/F6 and NP-C8/F6. The organic and the aqueous layers were separated, the organic layer was washed with brine $(1 \times 20 \mathrm{~mL})$ and the nanoparticles were precipitated by addition of methanol to the organic phase. The turbid suspension was transferred into two centrifuge tubes and centrifuged for 30 minutes at $4500 \mathrm{rpm}$ at $15{ }^{\circ} \mathrm{C}$. The supernatant was discarded and the solid residue was dissolved in $1 \mathrm{~mL}$ of $\mathrm{CHCl}_{3}$; the nanoparticles were precipitated a second time by addition of methanol and recovered by centrifugation. After removal of the supernatant, the solid was washed with $\mathrm{MeOH}(3 \times 15 \mathrm{~mL})$ and acetone $(3 \times 15 \mathrm{~mL})$. The purified nanoparticles were subjected to selective extractions with $\mathrm{CHCl}_{3}$ and afterwards with hexane. The insoluble material eventually present was tested for solubility in hexafluorobenzene. All of the fractions were characterised using ${ }^{1} \mathrm{H}$ NMR, UV-VIS, TGA, TEM. Characterization data are reported in Table S4 for NP-brC12/F6 and Table S6 for NP-C8/F6. $\dagger$

Nanoparticles NP-C16/F6. The organic and the aqueous layers were separated and the organic layer was washed with brine $(1 \times 20 \mathrm{~mL})$. The nanoparticles were precipitated by addition of methanol. The turbid suspension was transferred into two centrifuge tubes and centrifuged for 3 minutes at $4500 \mathrm{rpm}$ at $15{ }^{\circ} \mathrm{C}$. The supernatant was discarded and the precipitate was washed with methanol. The crude nanoparticle preparation was recovered by centrifugation. The nanoparticles were dissolved in $1.0 \mathrm{~mL}$ of $\mathrm{CHCl}_{3}$, the solvent was removed in an argon stream and the residue was washed with methanol $(7 \times 15 \mathrm{~mL})$. The purified 
nanoparticles were subjected to selective extractions with $\mathrm{CHCl}_{3}$ and afterwards with hexane. The insoluble material eventually present was tested for solubility in hexafluorobenzene. All of the fractions were characterised using ${ }^{1} \mathrm{H}$ NMR, UV-VIS, TGA, TEM. Characterization data are reported in Table S8. $\dagger$

\section{Acknowledgements}

This work was partly supported by the University of Trieste (FRA 2014, FRA 2015), FIRB 2011 Prot. RBAP11ETKA and MIUR: project MULTINANOITA. TEM images were recorded at the Microscopy Facility of the Department of Molecular Sciences and Nanosystems of the University $\mathrm{Ca}^{\prime}$ Foscari of Venezia or at the Electron Microscopy Facility of the University of Trieste (with the assistance of Mr Claudio Gamboz and Dr Paolo Bertoncin).

\section{References and notes}

1 P. Pengo and L. Pasquato, J. Fluorine Chem., 2015, 177, 2-10.

2 C. Gentilini, F. Evangelista, P. Rudolf, P. Franchi, M. Lucarini and L. Pasquato, J. Am. Chem. Soc., 2008, 130, 15678-15682.

3 T. Nishio, K. Niikura, Y. Matsuo and K. Ijiro, Chem. Commun., 2010, 46, 89778979.

4 K. Niikura, N. Iyo, Y. Matsuo, H. Mitomo and K. Ijiro, ACS Appl. Mater. Interfaces, 2013, 5, 3900-3907.

5 K. Niikura, N. Iyo, T. Higuchi, T. Nishio, H. Jinnai, N. Fujitani and K. Ijiro, J. Am. Chem. Soc., 2012, 134, 7632-7635.

6 M. Boccalon, S. Bidoggia, F. Romano, L. Gualandi, P. Franchi, M. Lucarini, P. Pengo and L. Pasquato, J. Mater. Chem. B, 2014, 3, 432-439.

7 M. Boccalon, P. Franchi, M. Lucarini, J. J. Delgado, F. Sousa, F. Stellacci, I. Zucca, A. Scotti, R. Spreafico, P. Pengo and L. Pasquato, Chem. Commun., 2013, 49, 8794-8796.

8 C. Gentilini, P. Franchi, E. Mileo, S. Polizzi, M. Lucarini and L. Pasquato, Angew. Chem., Int. Ed., 2009, 48, 3060-3064.

9 P. Posocco, C. Gentilini, S. Bidoggia, A. Pace, P. Franchi, M. Lucarini, M. Fermeglia, S. Pricl and L. Pasquato, ACS Nano, 2012, 6, 7243-7253.

10 M. P. Krafft and J. G. Riess, Chem. Rev., 2009, 109, 1714-1792.

11 J. D. Dunitz, ChemBioChem, 2004, 5, 614-621.

12 L. D. Zarzar, V. Sresht, E. M. Sletten, J. A. Kalow, D. Blankschtein and T. M. Swager, Nature, 2015, 518, 520-524.

13 N. C. Yoder, V. Kalsani, S. Schuy, R. Vogel, A. Janshoff and K. Kumar, J. Am. Chem. Soc., 2007, 129, 9037-9043.

14 P. Long and J. Hao, Adv. Colloid Interface Sci., 2012, 171-172, 66-76.

15 S. De Feyter and F. C. De Schryver, J. Phys. Chem. B, 2005, 109, 4290-4302.

16 M. Kadi, P. Hansson, M. Almgren and I. Furò, Langmuir, 2002, 18, 9243-9249.

17 V. Percec, M. R. Imam, M. Peterca and P. Leowanawat, J. Am. Chem. Soc., 2012, 134, 4408-4420.

18 V. Percec, M. Glodde, T. K. Bera, Y. Miura, I. Shiyanovskaya, K. D. Singer, V. S. K. Balagurusamy, P. A. Heiney, I. Schnell, A. Rapp, H.-W. Spiess, S. D. Hudsonk and H. Duank, Nature, 2002, 419, 384-862. 
19 V. Percec, G. Johansson, G. Ungar and J. Zhou, J. Am. Chem. Soc., 1996, 118, 9855-9866.

20 B. Bilgicüer, X. Xing and K. Kumar, J. Am. Chem. Soc., 2001, 123, 11815-11816.

21 B. C. Buer and E. N. G. Marsh, Protein Sci., 2012, 21, 453-462.

22 A. Centrone, E. Penzo, M. Sharma, J. W. Myerson, A. M. Jackson, N. Marzari and F. Stellacci, Proc. Natl. Acad. Sci. U. S. A., 2008, 105, 9886-9891.

23 A. Verma, O. Uzun, Y. H. Hu, Y. Hu, H. S. Han, N. Watson, S. L. Chen, D. J. Irvine and F. Stellacci, Nat. Mater., 2008, 7, 588-595.

24 A. Verma and F. Stellacci, Small, 2010, 6, 12-21.

25 R. C. Van Lehn, P. U. Atukorale, R. P. Carney, Y.-S. Yang, F. Stellacci, D. J. Irvine and A. Alexander-Katz, Nano Lett., 2013, 13, 4060-4067.

26 S. Jiang, Q. Chen, M. Tripathy, E. Luijten, K. S. Schweizer and S. Granick, Adv. Mater., 2010, 22, 1060-1071.

27 A. Ghosh, S. Basak, B. H. Wunsch, R. Kumar and F. Stellacci, Angew. Chem., Int. Ed., 2011, 50, 7900-7905.

28 A. M. Jackson, J. W. Myerson and F. Stellacci, Nat. Mater., 2004, 3, 330-336.

29 A. M. Jackson, Y. Hu, P. J. Silva and F. Stellacci, J. Am. Chem. Soc., 2006, 128, 11135-11149.

30 A. Centrone, Y. Hu, A. M. Jackson, G. Zerbi and F. Stellacci, Small, 2007, 3, 814817.

31 Q. K. Ong, J. Reguera, P. J. Silva, M. Moglianetti, K. Harkness, M. Longobardi, K. S. Mali, C. Renner, S. De Feyter and F. Stellacci, ACS Nano, 2013, 7, 85298539.

32 G. A. DeVries, M. Brunnbauer, Y. Hu, A. M. Jackson, B. Long, B. T. Neltner, O. Uzun, B. H. Wunsch and F. Stellacci, Science, 2007, 315, 358-361.

33 H. Kim, R. P. Carney, J. Reguera, Q. K. Ong, X. Liu and F. Stellacci, Adv. Mater., 2012, 24, 3857-3863.

34 Y. Hu, O. Uzun, C. Dubois and F. Stellacci, J. Phys. Chem. C, 2008, 112, 62796284.

35 O. Uzun, Y. Hu, A. Verma, S. Chen, A. Centrone and F. Stellacci, Chem. Commun., 2008, 196-198.

36 C. Singh, P. K. Ghorai, M. A. Horsch, A. M. Jackson, R. G. Larson, F. Stellacci and S. C. Glotzer, Phys. Rev. Lett., 2007, 99, 226106.

37 I. C. Pons-Siepermann and S. C. Glotzer, ACS Nano, 2012, 6, 3919-3924.

38 C. Singh, A. M. Jackson, F. Stellacci and S. C. Glotzer, J. Am. Chem. Soc., 2009, 131, 16377-16379.

39 A. Santos, C. Singh and S. C. Glotzer, Phys. Rev. E, 2010, 81, 011113.

40 R. P. Carney, G. A. DeVries, C. Dubois, H. Kim, J. Y. Kim, C. Singh, P. K. Ghorai, J. B. Tracy, R. L. Stiles, R. W. Murray, S. C. Glotzer and F. Stellacci, J. Am. Chem. Soc., 2008, 130, 798-799.

41 H. Singh, Y. Hu, B. P. Khanal, E. R. Zubarev, F. Stellacci and S. C. Glotzer, Nanoscale, 2011, 3, 3244-3250.

42 X. Liu, M. Yu, H. Kim, M. Mameli and F. Stellacci, Nat. Commun., 2012, 3, 1182.

43 F. Manea, C. Bindoli, S. Polizzi, L. Lay and P. Scrimin, Langmuir, 2008, 24, 4120-4124.

44 G. Guarino, F. Rastrelli, P. Scrimin and F. Mancin, J. Am. Chem. Soc., 2012, 134, 7200-7203.

45 R. S. Ingram, M. J. Hostetler and R. W. Murray, J. Am. Chem. Soc., 1997, 119, 9175-9178. 
46 R. Da Costa, M. Jurisch and J. Gladysz, Inorg. Chim. Acta, 2008, 361, 3205-3214.

47 T. Shimizu, T. Teranishi, S. Hasegawa and M. Miyake, J. Phys. Chem. B, 2003, 107, 2719-2724.

48 K. M. Harkness, A. Balinski, J. A. McLean and D. E. Cliffel, Angew. Chem., Int. Ed., 2011, 50, 10554-10559.

49 A. Stewart, S. Zheng, M. R. McCourt and S. E. J. Bell, ACS Nano, 2012, 6, 37183726.

50 M. J. Hostetler, J. E. Wingate, C.-J. Zhong, J. E. Harris, R. W. Vachet, M. R. Clark, J. D. Londono, S. J. Green, J. J. Stokes, G. D. Wignall, G. L. Glish, M. D. Porter, N. D. Evans and R. W. Murray, Langmuir, 1998, 14, 17-30.

51 P. J. Krommenhoek, J. Wang, N. Hentz, A. C. Johnston-Peck, K. A. Kozek, G. Kalyuzhny and J. B. Tracy, ACS Nano, 2012, 6, 4903-4911.

52 V. H. Dalvia and P. J. Rosskya, Proc. Natl. Acad. Sci. U. S. A., 2010, 107, 1360313607.

53 Y. Wang, O. Zeiri, A. Neyman, F. Stellacci and I. A. Weinstock, ACS Nano, 2012, 6, 629-640.

54 M. Brust, M. Walker, D. Bethell, D. J. Schiffrin and R. Whyman, J. Chem. Soc., Chem. Commun., 1994, 801-802. 